\title{
Der Einsatz des Europäischen Portfolios der Sprachen im Englischunterricht - Zentrale Ergebnisse einer quasi-experimentellen Feldstudie
}

\author{
Julia Dinn (Aachen/Deutscbland)
}

\section{Stand der Forschung und Erkenntnisdesiderata der Studie}

Gemäß dem Barcelona-Ziel sollen Europäerinnen und Europäer neben ihrer Erstsprache zwei weitere Fremdsprachen unabhängig von ihrer Bildungsbiographie erlernen. „Jeder sollte drei Gemeinschaftssprachen beherrschen“ (Europäische Kommission 1995: 62). Um diese sprachenpolitisch geforderte individuelle Mehrsprachigkeit umzusetzen, wird besonders auf zwei zentrale sprachenpolitische Instrumente des Europarats, den Gemeinsamen Europäischen Referenzrahmen (GER) und das etwa zeitgleich publizierte Europäische Portfolio der Sprachen (ESP), gesetzt.

Portfolios sind in aller Munde: Bei einer Google-Suchanfrage erhält der Begriff „Portfolio“ aktuell 618.875.000 mehr Suchtreffer als der Begriff „Fremdsprachendidaktik“. Im Folgenden werden die Bedeutung und Reichweite einzelner zentraler Ergebnisse einer 2017 abgeschlossenen Dissertation zum Europäischen Portfolio der Sprachen im Fremdsprachenunterricht mit dem Titel „Europäische Sprachenund Bildungspolitik: eine quasi-experimentelle Feldstudie zum Europäischen Portfolio der Sprachen“ erörtert (vgl. Dinn 2017). Zunächst werden das diesbezüglich relevante Erkenntnisinteresse sowie entsprechende Forschungsdesiderata dargestellt. 
Das Europäische Portfolio der Sprachen wurde vom Europarat verabschiedet und besteht aus drei wesentlichen Elementen, der Sprachenbiographie ${ }^{1}$, dem Sprachendossier $^{2}$ und dem Sprachenpass ${ }^{3}$. Laut der Entwicklerinnen und Entwickler soll das Lehr-Lern-Instrument den Sprachlernprozess begleiten und unterstützen, (außer)schulisches Lernen dokumentieren, zur Reflexion anregen, somit zur Herausbildung eines interkulturellen Bewusstseins beitragen und ein Sprachlernbewusstsein schaffen. ${ }^{4}$ Im Sprachenpass und der Sprachenbiographie des Portfolios sind an die Formulierungen aus dem Referenzrahmen angelehnte Selbsteinschätzungsitems implementiert, mittels derer die Lernenden in wiederkehrenden Abständen im Unterricht ihren aktuellen Sprachstand einschätzen sollen. Neben der Ausbildung von Selbsteinschätzungskompetenz hat das ESP die Verbreitung des GER mit seinen definierten Sprachkompetenzniveaus im unterrichtspraktischen Einsatz als Ziel (vgl. Schneider \& North 2000: 179-184). Paulson, Paulson und Meyer sind optimistisch, was die generelle Wirkungsweise von Portfolios angeht: „Portfolios can become a window into the students' heads, a means for both staff and students to understand the educational process at the level of the learner" (Paulson et al. 1991: 62). Eine Untersuchung der bundesdeutschen Gymnasialcurricula für Englisch als erste bzw. zweite Fremdsprache zeigte, dass der Einsatz des Europäischen Portfolios zur Entwicklung einer adäquaten Selbsteinschätzungskompetenz auch dort vielfach postuliert und in der Konsequenz eingefordert wird (vgl. Dinn 2017: 95-102). Ziel war es nun, näher zu betrachten und zu erheben, inwiefern sich die Effekte vom am GER orientierten ESP auf den modernen Fremdsprachenunterricht quantifizieren und qualifizieren lassen.

Die Idee des Portfolios als Sammelstelle ist bereits Jahrhunderte alt (vgl. Häcker 2011b), wesentlich jünger ist sein Einsatz im Fremdsprachenunterricht, der sich angesichts seiner Komplexität als noch wenig empirisch beforscht beschreiben lässt (vgl. Quetz 2011: 33-48). Die u.a. von Häcker beanstandete Theoriearmut des unterrichtlichen Portfolioansatzes legt eine weitere Beforschung des Lehr-Lerninstruments nahe, um dessen Einsatz möglichst optimal zu gestalten (vgl. Häcker 2011a: 6). Mitunter wird auch Kritik an der bisherigen Begleitforschung geübt, die als unreflektiert und distanzlos eingeschätzt wird, da sie nur ,vereinzelt“ und mit „ohnehin motivierten Lehrpersonen“ erfolgt, die häufig aus ihrer eigenen Erfahrung berichten (vgl. Quetz 2011: 33f.). Erkenntnisse basierend auf Aktionsforschung sowie der Einsatz des Portfolios durch Forschende selbst oder durch bereits portfolio-erfahrene Lehrende ermöglichen eine nur bedingte Übertragbar-

\footnotetext{
1 https://www.schulministerium.nrw.de/docs/Schulsystem/Unterricht/Lernbereiche-und-Faecher/ Fremdsprachen/Kontext/Europaeisches-Portfolio-derSprachen/biografie.pdf [10.12.2018].

2 https://www.schulministerium.nrw.de/docs/Schulsystem/Unterricht/Lernbereiche-und-Faecher/ Fremdsprachen/Kontext/Europaeisches-Portfolio-der-Sprachen/dossier.pdf [10.12.2018].

3 https://www.schulministerium.nrw.de/docs/Schulsystem/Unterricht/Lernbereiche-und-Faecher/ Fremdsprachen/Kontext/Europaeisches-Portfolio-der-Sprachen/sprachenpass.pdf [10.12.2018].

${ }^{4}$ Vgl. Schneider \& North (2000); Für vertiefende Betrachtungen zum Konzept des Portfolios siehe Dinn 2017: 80-106.
} 
keit und lassen essentielle Fragen zum Unterricht mit dem Portfolio unbeantwortet. Zudem wurden bislang ebenfalls noch nicht in ausreichendem Maß die langfristigen unterrichtlichen Veränderungen mittels Langzeitstudien beforscht (vgl. Häcker 2011a: 309). Letztlich fehlt es außerdem an fundierten Anhaltspunkten, wie genau und weshalb der Verbreitungsgrad des ESP in Europa laut der zuletzt dazu veröffentlichten Zahlen von Schärer (2008) hinter den Erwartungshaltungen zurückbleibt (vgl. Little et al. 2011: 5).

Betrachtet man die möglichen Effekte des ESP-Einsatzes im Unterricht, ist insbesondere der Aspekt der Selbsteinschätzung von Bedeutung. Das Ranking der umfangreichen Meta-Studie von Hattie (2015: 433) mit insgesamt 138 untersuchten Einflussgrößen sowie deren Effektstärken weist den Aspekt der Selbsteinschätzung des eigenen Leistungsniveaus als stärksten Einflussfaktor auf schulischen Lernerfolg aus. Auch Oscarson (1989: 3-5) benennt positive Effekte einer adäquaten Selbsteinschätzung, die sich auf den Fremdsprachenlernenden auswirken können, gleichwohl wird angemerkt, dass weitere empirische Validierungen von Nöten sind. Butler (2016) führt ebenso an, dass zuweilen für den Fremdsprachenunterricht zu wenige empirische Nachweise vorliegen, inwiefern die Selbsteinschätzung ein zutreffendes Abbild der lernersprachlichen Kompetenz darstellt: „However, we still have only a limited understanding on how SA [self assessment, J.D.] can best be used to facilitate children's learning“" (Butler 2016: 292).

Die Auswirkungen der Arbeit mit dem ESP auf die Korrektheit der Selbsteinschätzung (Selbsteinschätzungsadäquanz) beruhen vielfach auf weitestgehend unhinterfragten und unbeforschten Annahmen. Unter der Annahme einer grundlegenden Erlernbarkeit von Selbsteinschätzungskompetenz werden die Zielerwartungen in den Portfolios selbst artikuliert: „Das Einschätzen der eigenen Leistungen und Lernfortschritte ist lernbar, wenn man die richtigen Hilfsmittel dafür hat. In diesem Teil der Sprachen-Biografie könnt ihr lernen, wie man sich selbst beim Sprachenlernen überprüfen und Fortschritte einschätzen kann" (Europäisches Portfolio der Sprachen akk. Modell 46, Anhang, 2003: 21) sowie „Die kontinuierliche Arbeit am Europäischen Sprachenportfolio führt zu immer größerer Kompetenz, sich realistisch einzuschätzen" (akkreditiertes Modell 77.2006, Leitfaden 2008: 16)..$^{5}$

Augenscheinlich wird von Portfolioarbeit angenommen, dass sie zu einer Verbesserung der Selbsteinschätzungskompetenz beiträgt. Die einzig bekannte Studie, die dem in Teilen nachgeht, ist von Poppi \& Radighieri (2009); sie bezieht sich jedoch auf den universitären Kontext und verfolgt nur einen mehrwöchigen Ansatz. Im Kontext fremdsprachlichen Lernens mit dem ESP ist eine empirische kontrastive Erhebung von objektiver Lernleistungs- und subjektiv geprägter

\footnotetext{
${ }^{5}$ Solche optimistischen Einschätzungen bzw. Annahmen werden vielfach in der Portfolioforschungsliteratur wiedergegeben, vgl. dazu auch Bellingrodt (2011), Flächer (2011), Häcker (2011a), Little et al. (2011), Plötzleitner (2011) und Becker (2013).
} 
Selbstbeurteilung notwendig (vgl. Schneider \& North 2000, Vogt 2011) und durchgeführt worden.

Die beschriebene Forschungsarbeit umfasst u.a. die Durchführung einer einjährigen quasi-experimentellen Feldstudie. Dabei wurde der konkrete unterrichtspraktische Einsatz des ESP u.a. auch hinsichtlich des Effektes des Portfolioeinsatzes auf die Adäquanz der Selbsteinschätzung analysiert, um jene vorliegende Forschungslücke zu schließen. Des Weiteren wurde die bislang ebenfalls wenig beforschte unterrichtliche Verbreitung des GER im Rahmen der Dissertation untersucht. Die Ergebnisse der Studie liefern Anhaltspunkte, inwiefern die Zielsetzungen europäischer Sprachen- und Bildungspolitik konkret im fremdsprachlichen Lehr-/Lernkontext umgesetzt werden.

\section{Instrumentarien und Aufbau der Studie}

Für die Untersuchung wurde das europäische Portfolio der Sprachen über ein gesamtes Schuljahr hinweg im Englischunterricht sowohl an Gymnasien als auch an Gesamtschulen in der Sekundarstufe I eingesetzt. Dabei setzten unterrichtserfahrene und zugleich jedoch portfoliounerfahrene Lehrpersonen innerhalb ihrer bereits bestehenden Lerngruppen das Portfolio im Englischunterricht ein. Die Lehrkräfte erhielten eine grundlegende Einführung zu den Eigenschaften und Einsatzmöglichkeiten des Portfolios. Die konkrete und unterrichtspraktische Umsetzung wurde individuell von den Lehrenden an die jeweilige Lerngruppe und den Lernstand adaptiert.

Aus der Fülle der 141 vom Europarat bis 2014 akkreditierten bzw. registrierten Portfolioversionen ist das vom nordrhein-westfälischen Ministerium für Schule und Weiterbildung empfohlene und bereitgestellte Portfolio (Modell 46.2003) verwendet worden. Die Prä-Post-Testung der Selbsteinschätzungskompetenz der Schülerinnen und Schüler unter Bezugnahme zu Unterrichtsaktivitäten wurde mittels der auf dem GER beruhenden Can-do-statements einer anderen PortfolioVersion (Modell 90.2007, vgl. Europarat 2018) durchgeführt, um Reproduktionsbzw. Erinnerungseffekten vorzubeugen. Die der Erhebung der Selbsteinschätzung gegenüber gestellte Sprachkompetenzmessung wurde durch die von Cambridge English Language Assessment der University of Cambridge freundlicherweise bereitgestellten Sprachlesetests ermittelt. Dabei handelt es sich jeweils um zwei Sets der Testreihen Movers (Young Learners), Key English Test for Schools und Preliminary English Test for Schools bzw. die sprachlichen Niveaus des GER A1, A2 und B1. Obgleich die Evaluation der Lesefähigkeit nur stellvertretend für die sprachliche Kompetenz in der englischen Sprache stand, entspricht dies für Studien im fremdsprachendidaktischen bzw. angewandt-linguistischen Kontext einer üblichen Vorgehensweise. Verschiedene Studien, darunter die Meta-Studie von Ross (1998), stellen die Selbsteinschätzung der Lesekompetenz als besonders valide heraus. 
Im Rahmen der Längsschnittstudie wurden zum ersten Messzeitpunkt Daten von 480 Schülerinnen und Schülern sowie 16 Lehrpersonen an fünf Schulen erhoben. Die Stichprobe teilte sich in acht Experimentalgruppen und dem Parallelisierungsprinzip entsprechend acht Kontrollgruppen auf. Aufgrund der Panelmortalität, also der Divergenz der Anzahl der Datensätze von Zeitpunkt 1 und 2 (T1 und T2), wurden im Anschluss an die Anwendung des Portfolios Daten von 260 Lernenden, fünf Experimental- und fünf Kontrollgruppen sowie zehn Lehrenden an vier Schulen gesammelt und ausgewertet. Es handelte sich bei den Lerngruppen um 6., 7. Und 9. Klassen. Die Longitudinalstudie widmete sich sowohl quantitativen als auch qualitativen Datensätzen, die über papierbasierte Fragenbögen lehrerund schülerseitig sowie über teilstandardisierte Leitfadeninterviews mit den Experimental-Lehrpersonen ermittelt wurden. Die abgeschlossene Panelstudie ermittelte insgesamt 320 Variablen aus 760 Lernenden- und Lehrenden-Fragebögen.

Bei der empirischen Erhebung wurde der Einfluss von Portfolioarbeit auf verschiedene Lernervariablen (darunter der Korrektheitsgrad der Selbsteinschätzung) evaluiert. Ferner wurde untersucht, ob der Effekt des Portfolios bei allen untersuchten Lernenden im selben Ausmaß auftritt. Des Weiteren wurde betrachtet, ob sich die Wahrnehmung des ESP von Lernenden und Lehrenden im Zuge des Portfolioeinsatzes veränderte. Schließlich kam es zu einer Zusammenführung der Sichtweisen von Lernenden und Lehrenden hinsichtlich des (Nicht-)Gelingens von Portfolioarbeit.

\section{Ergebnisse der Studie}

\subsection{Evaluierung der Messinstrumente}

Die zur Testung der Selbsteinschätzungskompetenz verwendeten items des Portfolio-Modells 90.2007 (vgl. Europarat 2018) wurden statistischen Untersuchungen unterzogen. Um sicherzustellen, dass die Messungen reliabel waren und die Testinstrumente valide gemessen haben, was sie sollten, wurden die Standardfehler, Cronbachs Alpha sowie die Faktoren explorativ analysiert. Die explorativen Faktorenanalysen der zu allen Zeitpunkten durchgeführten Selbsteinschätzungstests erzielten durchschnittlich eher mittelmäßige Ergebnisse: Insbesondere manche der Selbsteinschätzungs-Deskriptoren wiesen latente, also über die beiden Messzeitpunkte anhaltende Schwierigkeiten auf, da die Ergebnisse dieser Deskriptoren bei der Messwiederholung wenig stabil waren. Auch bei der Betrachtung der Cronbach-Werte für die interne Konsistenz der Skala fallen Unregelmäßigkeiten auf: Die Selbsteinschätzungsitems für die 6. Und die 9. Klassen schneiden in akzeptablem Maße ab. Jedoch wurden die Cronbach-Werte für die 7. Klasse zu beiden Messzeitpunkten als eher niedrig interpretiert. Die Werte der Standardfehler waren unauffällig. Die Formulierungen der items, die an die Formulierungen des GER 
angelehnt sind, lassen die Vermutung zu, dass die Formulierungen des Referenzrahmens überdacht werden müssten.

Die oben genannten sowie weitergehende Analysen zur Validität und Reliabilität wurden auch für die Testung der Leseleistung im Englischen durchgeführt. Die berechneten Maße (Cronbachs Alpha, Standardabweichungen, explorative Faktorenanalyse) ließen keinerlei größere Auffälligkeiten oder Abweichungen feststellen. Zur Sicherstellung der gleichmäßigen Verteilung der Schwierigkeitsgrade der einzelnen items wurden in Anlehnung an Bühner (2011: 219-235) die Histogramme sowie die Mittelwerte der Lesetests auf Unregelmäßigkeiten überprüft. Alle zur Bestimmung der Testqualität herangezogenen Kenngrößen ergaben, dass die Kriterien stabiler Lesetests hinreichend erfüllt wurden. Die Auswertung der standardisierten Lesetests mit geschlossenen Aufgabenformaten und vorgegebenen Antwortoptionen verhalf außerdem durch die geringfügigen Interpretationsspielräume zu einer hohen Auswertungsobjektivität. ${ }^{6}$

\subsection{Einfluss des Portfolios auf die Selbsteinschätzung}

Die Ergebnisse der Selbsteinschätzungstests wurden den Lesetestergebnissen fallbezogen gegenübergestellt, um herauszufinden, ob eine sich von T1 zu T2 zum Positiven verändernde Selbsteinschätzungskompetenz mit dem Einsatz des Portfolios korreliert. Die Ergebnisse der Versuchsgruppen wurden demzufolge mit den Ergebnissen der Kontrollgruppen, die keinen Portfolioeinsatz im Unterricht erfuhren, verglichen. Hierzu wurden Signifikanz-, Effektstärke- und bivariate logistische Regressionsanalysen durchgeführt. Die präsentierte Datenlage legte nahe, dass ein Effekt eines Portfolios auf die Adäquanz der Selbsteinschätzung des eigenen fremdsprachlichen Leistungsstandes mittels dieser Studie nicht nachgewiesen werden konnte (Fischer=1,000; Cramérs V=0,010 bzw. Fischer=0,481; Cramérs $\mathrm{V}=0,050) .^{7}$

Auch andere lernerbezogene Variablen wurden im Kontext der Portfoliostudie untersucht: Weder ein erhöhter Lernzuwachs der Portfoliolernerinnen und -lerner noch Zusammenhänge zwischen einer sich erfolgreich entwickelnden Selbsteinschätzungskompetenz und individuellen Faktoren (Geschlecht, Portfoliovorerfahrung etc.) stellten sich heraus. Die schriftliche Befragung der Schülerinnen und Schüler ergab zwar, dass etwas mehr als die Hälfte der Portfoliolernerinnen und -lerner grundsätzlich eine Verbesserung der subjektiv konstatierten Selbsteinschätzungsadäquanz annahm. Die Gegenüberstellung der erhobenen Werte der Selbsteinschätzungskompetenz mit den Testergebnissen bestätigte diesen Eindruck jedoch nicht.

\footnotetext{
${ }^{6}$ Für detaillierte Ausführungen zur empirischen Betrachtung der im Rahmen der Studie verwendeten Erhebungsinstrumente siehe Dinn (2017: 144-157).

${ }^{7}$ Für detaillierte Ausführungen zur empirischen Betrachtung des Zusammenhangs zwischen dem Portfolioeinsatz und der Selbsteinschätzung siehe Dinn (2017: 196-245).
} 
Die im Kontext dieses Studiendesigns konstatierte Abwesenheit positiver Einflüsse durch die Portfolioarbeit auf die Ausbildung einer adäquaten Selbsteinschätzung stellt aufgrund der forschungsseitigen Wirksamkeitserwartung des Portfolioeinsatzes auf die Selbsteinschätzungsadäquanz eines der zentralen Resultate der Studie dar.

\subsection{Eindrücke der Studienteilnehmer zur Portfolioarbeit}

Die Evaluation der Eindrücke erfolgte einerseits mittels der Auswertung der Lernenden- und Lehrenden-Fragebögen. Außerdem wurden Befragungen der Lehrpersonen, die das Portfolio im Unterricht eingesetzt hatten, in Form von teilstandardisierten Experten-Interviews durchgeführt. Zur Dokumentation der LeitfadenInterviews zu den gemachten Erfahrungen wurden wegen der Konzentration auf inhaltliche Schwerpunkte kondensierte, zusammenfassende Protokolle erstellt (vgl. Mayring 2002: 96). Neben inhaltlichen Analysen, beispielsweise mittels induktiver Kategorienbildung für die erläuterten Vorteile des Portfolioeinsatzes, wurden u.a. auch Frequenzanalysen, etwa für die Zählung genannter Argumente, durchgeführt (vgl. Bortz \& Döring 2006: 185, Mayring 2015: 111, Kuckarts et al. 2008).

Starke signifikante Effekte bei den Korrelationen ${ }^{8}$ (Fisher=0,000; Cramérs V= $0,653)$ der Bewertungsmuster und dem Wunsch nach Fortführung oder Beendigung des Portfolioeinsatzes konnten herausgearbeitet werden. Die Portfolionutzerinnen und -nutzer, die dem Instrument eine bessere Note gaben (Notenspektrum „sehr gut" bis „befriedigend“ im Gegensatz zu ,ausreichend“ bis „,ungenügend“), wünschten auch eher weiterhin mit dem Portfolio im Unterricht arbeiten zu können. Ebenfalls starke signifikante Zusammenhänge (Fisher $=0,000$, Cramérs $\mathrm{V}=0,525)$ sind bei der Betrachtung der Variablen bezüglich des Wunsches nach Fortführung sowie der wahrgenommenen Verbesserung der Selbsteinschätzungskompetenz zu berichten gewesen. Wer eher eine positive Wirkung des Portfolios auf die Selbsteinschätzungskompetenz annahm, wünschte auch den weiteren Einsatz im Englischunterricht.

Betrachtete man den Zusammenhang zwischen den lernerseitigen Portfoliobewertungen und der wahrgenommenen Verbesserung der Einschätzung der Englischkenntnisse, so lagen hier signifikante mittlere Zusammenhänge vor (Fisher= 0,000 , Cramérs V =0,401). Wer annahm, die eigene Selbsteinschätzungskompetenz mittels des Portfolios verbessert haben zu können, bewertete das Portfolio auch signifikant besser.

Die Datenlage legt offen, dass die Lernenden, die das Portfolio eher negativ bewerteten und keine Fortsetzung der Portfolioarbeit wünschten, zugleich nicht glaubten, dass ihnen die Arbeit mit dem Instrument bei der Weiterentwicklung ihrer Selbsteinschätzungskompetenz geholfen hatte. Durch diese Studie wurde ein

\footnotetext{
8 Basierend auf der Art der vorliegenden Variablen und der Anzahl der Fälle wurde das Signifikanzniveau mittels Fishers exaktem Test und das Effektstärkemaß mittels Cramérs V ermittelt.
} 
Zusammenhang zwischen Bewertungsmustern einerseits und Wirksamkeitserwartungen andererseits belegt. Den schriftlichen Aussagen der Teilnehmerinnen und Teilnehmer zufolge hatten diejenigen Gruppen, die der Portfolioarbeit ein schlechteres Zeugnis ausstellten, sich während des Schuljahrs auch vergleichsweise weniger intensiv mit dem Instrument auseinandergesetzt als die anderen Gruppen. Auch das signifikante Ergebnis der Studie von Becker $(2013: 142,162)$ weist eine Korrelation zwischen dem intensiven Einsatz des Portfolios im Unterricht und dem Wunsch nach Fortsetzung der ESP-Arbeit aus.

Nach dem Nutzen des Portfolios befragt, hatten die Lehrkräfte ebenso wie die Schülerinnen und Schüler sehr unterschiedliche Wahrnehmungen und Bewertungen. Der für die Einführung und Anwendung des Portfolios notwendige Zeitaufwand wurde beiderseits als deutlicher Nachteil des Portfolioansatzes herausgestellt. Die Datenlage zeigte bei den einzelnen Lerngruppen sehr homogene Reaktionen auf das Portfolio, die wiederum aber gruppenweise betrachtet sehr unterschiedlich waren und von Euphorie bis Enttäuschung reichten.

Eine Parallelität der Lehrendeneindrücke mit den Globaltrends der Gruppeneindrücke wurde deutlich. Die Erhebung zeigte, dass die von den Lehrerinnen und Lehrern wahrgenommene Wirksamkeit des Portfolios, der unterrichtspraktische Einsatz (wahrgenommen durch die Lernenden) sowie die lernerseitige Bewertung der Portfolioarbeit in einer Beziehung zueinander stehen. Die Daten verdeutlichten, dass bei der positiven bzw. negativen Rezeption des Portfolios Lernende und Lehrende zusammenwirkten. Aufgrund der Anonymisierung der Fragebögen der Lehrkräfte erfolgte keine Zuordnung zu den Daten der Lerngruppen. Es war jedoch beachtenswert, dass sowohl zwei Lehrer als auch zwei Lerngruppen dem Portfolio gegenüber eher reserviert und kritisch eingestellt waren. Die Lernenden dieser zwei Experimentalgruppen gaben darüber hinaus an, überwiegend weniger intensiv mit dem Portfolio vertraut gemacht worden zu sein. Sie bewerteten das Instrument tendenziell auch schlechter und wollten es mehrheitlich nicht weiter nutzen. Ebenso gaben zwei Lehrkräfte in den mündlichen Befragungen an, nach dem Abschluss der Studie nicht weiter mit dem Portfolio arbeiten zu wollen.

Die Daten der schriftlichen und mündlichen Befragung der Lernenden und Lehrenden offenbarten eine weitere Problematik: Nach den Eigenschaften des Portfolios und der darin enthaltenen GER-Elementen gefragt, ließen die gegebenen Antworten mitunter annehmen, dass das Konzept des Instruments seitens mancher Lehrpersonen und in der Folge dann auch seitens der Lernenden nicht vollumfänglich durchdrungen wurde. Obgleich die Einflussfaktoren auf die Ausbildung einer zutreffenden Selbsteinschätzungskompetenz breit gefächert sind, kann ein sich möglicherweise positiv darauf auswirkendes Instrument nur gezielt und gewinnbringend im Unterricht eingesetzt werden, wenn die Lehrpersonen und schließlich auch die Lernenden damit umgehen können. Um mit dem Instrument im Unterricht zu arbeiten und die Lernenden bei ihren Selbsteinschätzungsversuchen mittels des Portfolios zu beraten, wird u.a. Wissen um die GERKompetenzniveaus vorausgesetzt. Betrachtet man jedoch die eigenen Angaben, 
inwiefern und woher die Lehrkräfte der quasi-experimentellen Feldstudie ihr Wissen über die Ermittlung sprachlicher Kompetenzen erlangt haben, schienen sie im Wesentlichen über eine Assessment Literacy zu verfügen, die sich stark bis gänzlich aus der Praxis speist. Ähnliche Anhaltspunkte liefern auch Vogt und Tsagari (2014) in ihrer umfangreichen Lehrerbefragung. Auch mit dem Wissen über den GER und das Portfolioinstrument geht nicht automatisch einher, dass sich eine kompetenzorientierte Beratungs- und Diagnosefähigkeit einstellt. Ohne ein solides, theoretisch fundiertes und in der Praxis erprobtes Wissen sind die kompetenzorientierten Elemente des Portfolioansatzes, insbesondere im Bereich der Arbeit mit der Sprachenbiographie und dem Sprachenpass, schwer bis gänzlich nicht sinnvoll umsetzbar. Wenn die Lehrkräfte nicht anhand der GER-Niveaus bzw. -Deskriptoren grundsätzlich herausarbeiten können, wo die Stärken und Schwächen der ihnen anvertrauten Schülerinnen und Schüler liegen, können sie diese auch nicht zu ihrer Selbsteinschätzungskompetenz beraten.

\section{$4 \quad$ Fazit und Ausblick}

Da aktuell Lehrende bislang noch zu wenig im Umgang mit Portfolios und kompetenzorientierter Leistungsermittlung ausgebildet zu sein scheinen, können Schwierigkeiten erwachsen, ein Gespür für die Formulierungsschwächen des GER und den daraus resultierenden Erhebungsproblematiken von fremdsprachlicher Selbsteinschätzungskompetenz auszubilden. Es drängt sich die Frage auf, wie diese Lernenden dazu befähigt werden sollen, mit einem an diese Niveaubeschreibungen angelehnten Instrument $\mathrm{zu}$ arbeiten und sinnvoll Schlüsse für ihren eigenen Sprachlernprozess daraus zu ziehen. Dementsprechend naheliegend stellt sich im Lichte dieser Befunde auch die nicht existente Zunahme an adäquater Selbsteinschätzungskompetenz in den Portfoliogruppen im Vergleich zu ihren Kontrollgruppen dar.

Das ESP ist gekennzeichnet durch seine Spagatstellung zwischen Lernendenorientierung auf der einen Seite und Standardorientierung auf der anderen Seite (vgl. dazu auch Kolb 2007: 318). In der Sprachenbiographie und im Dossier werden die standardisierten GER-Referenzniveaus für Einschätzungen der einzelnen Kompetenzbereiche eingesetzt. Die Schülerinnen und Schüler orientieren sich hierbei also anhand einer vorgegebenen kriteriellen Norm im Gegensatz zur sozialen bzw. individuellen Norm. Demgegenüber sollen die Schülerinnen und Schüler im Dossier und in der Biographie ihren Lernprozess mittels ihrer Individualnorm zunächst planen und schließlich evaluieren. Für die Förderung von Standardisierung und Lernendenorientierung bietet das ESP durch seinen binär angelegten Ansatz zumindest theoretisch einen ersten Anlaufpunkt im Fremdsprachenunterricht. Die Handhabung in der Praxis erweist sich durch die vorliegende Studie als mindestens stark anleitungs- und einübungsbedürftig. Quetz äußert sich zur Selbsteinschätzungskompetenz wie folgt ebenso kritisch: 
Wir leben in einer Bildungslandschaft, die dem Gedanken des selbstbestimmten Lernens nicht gerade freundlich gesonnen ist. Wie soll da Portfolioarbeit zur Motivation der Lernenden beitragen, wenn man dazu auch noch feststellen muss, dass das ganze System der Selbstbeurteilung auf wackeligen Beinen steht und die Hoffnung, Sprachlernbewusstheit könne Lernen effektiver machen, unter Umständen auch nicht der Weisheit letzter Schluss ist? (Quetz 2011: 46)

Im Rahmen einer umfassenden Validierungsstudie sollten Beschreibungen des Portfolios sowie die Deskriptoren des GER optimiert werden. Lernende und Lehrende müssen sich auf die vielfach in den curricularen Vorgaben geforderten Instrumente verlassen können. Ein unsicheres Lehr-Lern-Instrument, das den Anschein von Messgenauigkeit erweckt und den Lernern beim Anwenden und Ausfüllen irrtümlich suggeriert, man habe ein fremdsprachliches Niveau (noch nicht) erreicht, erschwert bzw. gefährdet schlimmstenfalls den Entwicklungsprozess der subjektiven Selbsteinschätzungskompetenz (vgl. Dinn 2017: 261). Etwaige Änderungen und Ergänzungen der Referenzniveaubeschreibungen müssen dann mit in die Portfolios übernommen werden und dort zielgruppengerecht überführt und eingepasst werden, damit die Lernenden optimale und passgenaue Bezugsformulierungen haben, die statistischen Prüfungen besser standhalten.

Die Zentralität der Rolle der Lehrperson, die Schulung von Lehrkräften für den Unterrichtseinsatz des Portfolios und die Bedeutung institutioneller Begleitung für den Erfolg von Portfolioarbeit wurden anhand der Studie herausgearbeitet. Ein theoretisches Innovations- und Unterstützungspotential des ESP für den Fremdsprachenunterricht kann nur dann genutzt werden, wenn die Lehrenden fundiert geschult werden und ggf. aus der Lernendenperspektive eigene Erfahrungen mit kriterienorientierter, sprachlicher Selbsteinschätzung und Portfolionutzung sammeln.

Der zukünftige Umgang mit den problematischen Formulierungen des GER, die weitere wissenschaftliche Begegnung grundsätzlicher Einsatzherausforderungen des ESP sowie die Bewältigung des Mangels an im Umgang mit diesen Instrumenten ausgebildeten Lehrkräften sind gegenwärtige Herausforderungen der Fremdsprachendidaktik, der Angewandten Linguistik ebenso wie der Politik und bieten vielfältige Anknüpfungspunkte für weitere Forschungsprojekte. Das Companion Volume des GER, das seit 2014 entwickelt wurde, definiert neben der Aufnahme weiterer Fertigkeitsbereiche viele Niveaubeschreibungen und Zwischenniveaus umfangreicher. Es ist zu hoffen, dass die Aktualität und die größere Ausführlichkeit der Beschreibungen den Lernenden eine noch größere Hilfestellung als bisher sind.

Der von Ballweg \& Bräuer (2011) optimistisch verwendeten Überschrift „Portfolioarbeit im Fremdsprachenunterricht - Yes, we can!" fügt die Autorin daher hinzu „Maybe not yet!" 


\section{Literatur}

Ballweg, Sandra; Bräuer, Gerd (2011): Portfolioarbeit im Fremdsprachenunterricht - Yes, we can! In: Fremdsprache Deutsch 45/2, 3-11.

Becker, Carmen (2013): Portfolio als Baustein einer neuen Lernkultur - eine empirische Studie zur Implementierung des Europäischen Portfolios der Sprachen. Frankfurt/M.: Lang.

Bellingrodt, Lena Christine (2011): ePortfolios im Fremdsprachenunterricht: Empirische Studien zur Förderung autonomen Lernens. Frankfurt/M.: Lang.

Bortz, Jürgen; Döring, Nicola (2006): Forschungsmethoden und Evaluation für Humanund Sozialwissenschaftler. Berlin, Heidelberg: Springer Medizin.

Bühner, Markus (2010): Einführung in die Test-und Fragebogenkonstruktion. 3. Aufl. München: Pearson.

Butler, Yuko Goto (2016). Self-assessment of and for young learners' foreign language learning. In: Nikolov, Marianne (Hrsg.): Assessing Young Learners of English: Global and Local Perspectives. New York: Springer, 291-315.

Dinn, Julia (2017): Europäische Sprachen-und Bildungspolitik: Eine quasi-experimentelle Feldstudie zum Europäischen Portfolio der Sprachen. Aachen: RWTH Aachen University Press.

Europäische Kommission (1995): Weißbuch zur allgemeinen und beruflichen Bildung, Lebren und Lernen: Auf dem Weg zur kognitiven Gesellschaft. Luxemburg. https:/ / publications.europa.eu/en/publication-detail/-/publication/d0a8aa7a-53114eee-904c-98fa541108d8/language-de [01.03.2019].

Europarat (2018). Accredited and registered ELP by dates. https:/ /www.coe.int/en/ web/portfolio/accredited-and-registered-models-by-date - \{“11838743”:[7]\} [01.03.2019].

Flächer, Tina (2011): Portfolioarbeit im gymnasialen Fremdsprachenunterricht: Themenorientierter Unterricht mit dem Europäischen Portfolio der Sprachen. Frankfurt/M.: Lang.

Häcker, Thomas (2011a): Portfolio: Ein Entwicklungsinstrument für selbstbestimmtes Lernen. Eine explorative Studie zur Arbeit mit Portfolios in der Sekundarstufe I. 3. Aufl. Baltmannsweiler: Schneider Hohengehren.

Häcker, Thomas (2011b): Wurzeln der Portfolio-Arbeit: Woraus das Konzept erwachsen ist. In: Bruner, Ilse; Häcker, Thomas; Winter, Felix (Hrsg.): Das Handbuch Portfolio-Arbeit: Konzepte, Anregungen, Erfabrungen aus Schule und Lebrerbildung. 4. Aufl. Seelze: Kallmeyer/Klett, 27-32.

Hattie, John (2015): Lernen sichtbar machen - Überarbeitete deutschsprachige Ausgabe von Visible Learning. 3. Aufl. Baltmannsweiler: Schneider Hohengehren.

Kolb, Annika (2007): Portfolioarbeit: Wie Grundschulkinder ihr Sprachlernen reflektieren. Tübingen: Narr. 
Kuckartz, Udo; Dresing, Torsten; Rädiker, Stefan; Stefer, Claus (2008): Qualitative Evaluation: Der Einstieg in die Praxis. Wiesbaden: Verlag für Sozialwissenschaften.

Kultusministerium Hessen (2007): BLK-Verbundprojekt „Sprachen Lehren und Lernen als Kontinuum" Europäisches Portfolio der Sprachen ake.e. Modell 90.2007. http:/ /www.sprachenportfolio.de/ [01.03.2019].

Little, David; Goullier, Francis; Hughes, Gareth (2011): The ELP - the story so far (1991-2011): A bistory of the ELP. Straßburg: Europarat. https://rm.coe.int/CoERMPublicCommonSearchSe vices/DisplayDCTMContent?documentId=09000016804595a7 [01.03.2019].

Mayring, Philipp (2002): Einführung in die qualitative Sozialforschung: Eine Anleitung zu qualitativem Denken. Weinheim u. a.: Beltz.

Mayring, Philipp (2015): Qualitative Inhaltsanalyse: Grundlagen und Techniken. 12. Aufl. Weinheim u. a.: Beltz.

Oscarson, Mats (1989): Self-assessment of language proficiency: Rationale and applications. In: Language Testing 6/1, 1-13.

Paulson, F. Leon; Paulson, Pearl R.; Meyer, Carol A. (1991): What makes a portfolio a portfolio? In: Educational Leadership 48/5, 60-63. http:/ /www.ascd.org /ASCD/pdf/journals/ed_lead/el_199102_paulson.pdf [01.03.2019].

Plötzleitner, Elisabeth (2011): Reflektieren kann man lernen - Formblätter als Hilfe zur Selbsteinschätzung. In: Brunner, Ilse; Häcker, Thomas; Winter, Felix (Hrsg.): Das Handbuch Portfolio-Arbeit: Konzepte, Anregungen, Erfahrungen aus Schule und Lehrerbildung. 4. Aufl. Seelze: Kallmeyer/Klett, 96-111.

Poppi, Franca; Radighieri, Sara (2009): The role of the ELP and self-assessment in effective language learning. In: Gori, Frederica (Hrsg.): Il portfolio europeo delle lingue nee'universita intaliana. Trieste: Edizioni Universita di Trieste, 83-97.

Quetz, Jürgen (2011): Das Europäische Sprachenportfolio als Mittel zur Förderung des selbstgesteuerten Fremdsprachenlernens? In: Arntz, Reiner; Krings, Hans P.; Kühn, Bärbel (Hrsg.): Autonomie und Motivation: Erträge des 2. Bremer Symposions zum autonomen Fremdsprachenlernen. Bochum: AKS-Verlag, 33-48.

Ross, Steven J. (1998): Self-assessment in second language testing: A meta-analysis and analysis with experiential factors. In: Language Testing 15/1, 1-20.

Schärer, Rolf (2008): European Language Portfolio: Interim Report 2007. https:/ /rm.coe.int/CoERMPublicCommonSearchServices/DisplayDCTMContent?docu mentId $=09000016804595 a 6$ [01.03.2019].

Schneider, Günther; North, Brian (2000): Fremdsprachen können - was heisst das? Skalen zur Beschreibung, Beurteilung und Selbsteinschätzung der fremdsprachlichen Kommunikationsfähigkeit. Nationales Forschungsprogramm 33, Wirksamkeit unserer Bildungssysteme. Chur, Zürich: Rüegger.

Europäisches Portfolio der Sprachen akk. Modell 46.2003: Schroedel Diesterweg (2003). 
Vogt, Karin (2011): Fremdsprachliche Kompetenzprofile: Entwicklung und Abgleichung von GeR-Deskriptoren für Fremdsprachenlernen mit einer beruflichen Anwenderorientierung. Tübingen: Narr.

Vogt, Karin; Tsagari, Dina (2014): Assessment literacy of foreign language teachers: Findings of a European study. In: Language Assessment Quarterly 11/4, 374-402.

Thüringer Volkshochschulverband e.V. (2008): Leitfaden zum Europäischen Sprachenportfolio für Erwachsene zum Akk. Modell Nr. 77.2006. http://www.hueber.de /sixcms/media.php/36/sprachenportfolio_ene.pdf [01.03.2019]. 